\title{
Electromagnetic field in matter. Surface enhanced Raman scattering
}

\author{
M. Apostol ${ }^{1,2 *}$, S. Ilie ${ }^{2,3}$, A. Petrut ${ }^{3}$, \\ M. Savu ${ }^{2}$, and S. Toba ${ }^{3}$ \\ ${ }^{1}$ Department of Theoretical Physics, Institute of Atomic Physics, Magurele-Bucharest MG-6, POBox MG-35, Romania \\ ${ }^{2}$ MiraTechnologies Ltd, Teiul Doamnei 2, Bucharest \\ ${ }^{3}$ MiraTelecom, Grigorescu 13, 075100 Otopeni-Bucharest \\ *corresponding author, E-mail: apoma@theory . nipne.ro
}

\begin{abstract}
The polarization and magnetization degrees of freedom are included in the general treatment of the electromagnetic field in matter, and their governing equations are given. Particular cases of solutions are discussed for polarizable, non-magnetic matter, including quasi-static fields, surface plasmons, propagation, zero-point fluctuations of the eigenmodes, especially for a semi-infinite homogeneous body (half-space). The van der Waals-London-Casimir force acting between a neutral nano-particle and a half-space is computed and the response of this electromagnetically coupled system to an external field is given, with relevance for the surface enhanced Raman scattering.
\end{abstract}

\section{Introduction. General theory}

With usual notations the Maxwell equations in matter read

$$
\begin{gathered}
\operatorname{div} \mathbf{D}=4 \pi \rho_{0}, \operatorname{div} \mathbf{B}=0, \\
\operatorname{curl} \mathbf{E}=-\frac{1}{c} \frac{\partial \mathbf{B}}{\partial t}, \operatorname{curl} \mathbf{H}=\frac{1}{c} \frac{\partial \mathbf{D}}{\partial t}+\frac{4 \pi}{c} \mathbf{j}_{0},
\end{gathered}
$$

where $\mathbf{E}$ is the electric field, $\mathbf{D}$ is the electric displacement, $\mathbf{B}$ is the magnetic induction and $\mathbf{H}$ is the magnetic field; $\rho_{0}$ is the external charge density and $\mathbf{j}_{0}$ is the external current density (obeying the continuity equation $\left.\partial \rho_{0} / \partial t+\operatorname{div} \mathbf{j}_{0}=0\right)$. We have two independent Maxwell equations (1) (Faraday's and Maxwell-Ampere's equations) and four unknowns. In order to have a solution we introduce the quasi-phenomenological dielectric function $\varepsilon$ and magnetic permeability $\mu$, usually for the Fourier transforms. Apart from being unsatisfactory at the fundamental level, this procedure produce appreciable difficulties, especially with the finite size, inhomogeneous bodies.

Matter is polarizable, i.e. it consists of more-or-less mobile charges $q$, with mass $m$ and concentration $n$ (e.g., electrons), which move against a neutralizing background of quasi-rigid charges $-q$ (e.g., ions). A small displacement field $\mathbf{u}(t, \mathbf{r})$, which is a function of the time $t$ and position $\mathbf{r}$, generates an imbalance $\delta n=-n d i v \mathbf{u}$ in the density of these charges, a charge density $\rho=-n q d i v \mathbf{u}$ and a corresponding current density $\mathbf{j}=n q \dot{\mathbf{u}}$. It is easy to see that the polarization is $\mathbf{P}=n q \mathbf{u}$ (density of the dipole moments) and the electric displacement is now represented as
$\mathbf{D}=\mathbf{E}+4 \pi \mathbf{P}$. The displacement field obeys an equation of motion, which usually is Newton's equation

$$
m \ddot{\mathbf{u}}=q \mathbf{E}-m \omega_{c}^{2} \mathbf{u}-m \gamma \dot{\mathbf{u}}
$$

$\omega_{c}$ is a characteristic fequency (e.g., for bound charges) and $\gamma$ is a small damping coefficient. The magnetic term of the Lorentz force is usually absent in equation (2) (and the equation is non-relativistic), since the velocity of charges in matter is small, on one hand, and, on the other, the displacement $\mathbf{u}$ is sufficiently small to limit ourselves to linear terms only. This is the well-known Drude-Lorentz (plasma) model of polarizable matter.[1]-[3] The point is that the equation of motion (2) provides a third equation for the four unknowns: $\mathbf{E}, \mathbf{u}, \mathbf{B}$ and $\mathbf{H}$. It can be generalized in various ways, e.g. by including external fields.

Matter is also magnetizable. The continuity equation allows of a "magnetic" current $\mathbf{j}=c \cdot \operatorname{curl} \mathbf{M}$; as it is wellknown the magnetization $\mathbf{M}$ obeys the equation of motion of the angular momentum $[4,5]$

$$
\frac{d \mathbf{M}}{d t}=\frac{q}{2 m c} \mathbf{M} \times \mathbf{B} .
$$

The magnetic induction is now represented as $\mathbf{B}=\mathbf{H}+$ $4 \pi \mathbf{M}$. The "magnetic" current given above or the subsequent relationship between $\mathbf{B}$ and $\mathbf{H}$ are two well-known equivalent ways of introducing magnetization in Maxwell equations.[5]-[9] We have now four equations:

$$
\begin{gathered}
\operatorname{curl} \mathbf{E}=-\frac{1}{c} \frac{\partial \mathbf{B}}{\partial t}, \operatorname{curl} \mathbf{H}=\operatorname{curl}(\mathbf{B}-4 \pi \mathbf{M})= \\
=\frac{1}{c} \frac{\partial \mathbf{E}}{\partial t}+\frac{4 \pi}{c} n q \dot{\mathbf{u}}+\frac{4 \pi}{c} \mathbf{j}_{0}
\end{gathered}
$$

and equations (2) and (3) and four unknowns: E, u, B and M. These equations can provide the basis for treating the electromagnetic field in matter. Except for the important case of ferromagnetic (and related) matter, the usual matter is non-magnetic, so we may leave aside $\mathbf{M}$ and put $\mathbf{B}=\mathbf{H}$.

For the usual case of polarizable non-magnetic matter, we can find the plasmon and polariton eigenmodes, especially for infinite or semi-infinite (half-space) matter.[10, 11] We can thereby describe the propagation of electromagnetic field in matter, as well as the interaction of the electromagnetic field with finite-size bodies, both in the near-field (sub-wavelength, quasi-static) regime and the wave (radiation) zone. This can be done in complex situations, where 
various fields are present for bodies with various shapes, a subject of high interest for nano-plasmonics.[12] The plasmon and, respectively polariton eigenmodes are given by

$$
\begin{gathered}
\Omega_{1}=\omega_{L}=\sqrt{\omega_{c}^{2}+\omega_{p}^{2}}, \Omega_{2}(K) \simeq \sqrt{\omega_{L}^{2}+c^{2} K^{2}}, \\
\Omega_{3}(K) \simeq \omega_{c} c K /\left(\omega_{L}+c K\right)
\end{gathered}
$$

where $\omega_{p}=\left(4 \pi n q^{2} / m\right)^{1 / 2}$ is the plasma wavevector and $\mathbf{K}$ is the wavevector. For a half-space we get a surface plasmon-polariton mode

$$
\begin{gathered}
\Omega^{2}=\frac{2 A c^{2} k^{2}}{B+\sqrt{B^{2}-4 A c^{2} k^{2}}} \\
A=2 \omega_{c}^{2}+\omega_{p}^{2}, B=\omega_{c}^{2}+\omega_{p}^{2}+2 c^{2} k^{2}
\end{gathered}
$$

for $c^{2} k^{2}>\omega_{c}^{2}$, where $\mathbf{k}$ is the wavevector parallel with the surface; it goes from $\omega_{c}\left(c k=\omega_{c}\right)$ to $\sqrt{\omega_{c}^{2}+\omega_{p}^{2} / 2}(k \rightarrow$ $\infty)$. This mode is localized with respect to the direction perpendicular to the surface.

The zero-point energy (vacuum fluctuations) of the polarization eigenmodes leads to molecular forces like van der Waals-London-Casimir forces acting between macroscopic bodies.[13] The behaviour of the polarization eigenmodes in non-inertial motions may lead to interesting new effects.[14] The electromagnetic coupling between nanostructures can also be treated by this method, leading to plasmon transfer and resonances, or to electromagnetic forces with a resonant character.[15] The scattering of the electromagnetic waves by small particles or inhomogeneities, including the rough surface of a semi-infinite solid,[16] is also amenable to such a treatment.

\section{Quasi-static fields}

We write again the Maxwell equations in (non-magnetic, $\mathbf{H}=\mathbf{B}$ ) matter, with an explicit introduction of the polarization $\mathbf{P}$ :

$$
\begin{gathered}
\operatorname{div} \mathbf{E}=4 \pi \rho_{0}-4 \pi d i v \mathbf{P}, \operatorname{div} \mathbf{H}=0, \\
\operatorname{curl} \mathbf{E}=-\frac{1}{c} \frac{\partial \mathbf{H}}{\partial t}, \\
\operatorname{curl} \mathbf{H}=\frac{1}{c} \frac{\partial \mathbf{E}}{\partial t}+\frac{4 \pi}{c} \frac{\partial \mathbf{P}}{\partial t}+\frac{4 \pi}{c} \mathbf{j}_{0} ;
\end{gathered}
$$

the fields satisfy wave equations with sources (including polarization charges $-\operatorname{div} \mathbf{P}$ and currents $\partial \mathbf{P} / \partial t)$; as it is well known, it is convenient to introduce the potentials $\mathbf{A}$ and $\Phi$, such as $\mathbf{E}=-(1 / c) \partial \mathbf{A} / \partial t-\operatorname{grad} \Phi, \mathbf{H}=\operatorname{curl} \mathbf{A}$; these potentials satisfy the wave equations with sources, providing the Lorenz gauge $\operatorname{div} \mathbf{A}+(1 / c) \partial \Phi / \partial t=0$ is satisfied. We assume the quasi-static situation, i.e. we assume that the time variation is slow, such as to have $\omega / c \ll 1 / l$, and $v / c \ll 1$, where $\omega$ is the frequency, $l$ is the dimension of the bodies and $v$ is the velocity of the charges. Then, it is easy to see that from all the Maxwell equations only Gauss's equation

$$
\operatorname{div} \mathbf{E}=4 \pi \rho_{0}-4 \pi \operatorname{div} \mathbf{P}
$$

survives, and $\operatorname{cur} l \mathbf{E}=0$; which means that we may neglect A and keep only $\Phi$, such as $\mathbf{E}=-\operatorname{grad} \Phi$. This can also be seen directly from Kirchhoff's solutions of the wave equations for potentials. This is the quasi-static approximation (or even static, when there is not a time dependence); it is also termed the sub-wavelength or near-field regime. It is relevant for external $\rho_{0} \neq 0$ (or other external causes producing polarization charges). If this $\rho_{0}=0$ and no other external causes producing polarization charges are present, it is the next approximation which is relevant, corresponding to $\mathbf{j}_{0}$, with $\operatorname{div} \mathbf{j}_{0}=0$ and, of course , $\mathbf{A} \neq 0$ and $\Phi=0$. The former case is also called electrostatics (or quasi-electrostatics), the latter is magnetostatics, though, allowing for slow time dependence, they are both quasistatic.

It is convenient to work with an external (slowly varying in time) electric field $\mathbf{E}_{0}$ instead of the external charge density $\rho_{0}$ (a slow external magnetic field can also be present). With usual notations, the equation of motion (2) for the displacement $\mathbf{u}$ can be written as

$$
m \ddot{\mathbf{u}}=q\left(\mathbf{E}_{0}+\mathbf{E}\right)-m \omega_{c}^{2} \mathbf{u}-m \gamma \dot{\mathbf{u}},
$$

or, using the temporal Fourier transforms,

$$
\left(\omega^{2}-\omega_{c}^{2}+i \omega \gamma\right) \mathbf{u}=-\frac{q}{m}\left(\mathbf{E}_{0}+\mathbf{E}\right)
$$

where $\mathbf{E}$ is the internal (polarization) electric field, given by

$$
\operatorname{div} \mathbf{E}=4 \pi \rho=-4 \pi \operatorname{div} \mathbf{P}=-4 \pi n q \operatorname{div} \mathbf{u} ;
$$

we note that $\rho=-\operatorname{div} \mathbf{P}=-n q d i v \mathbf{u}$ denotes now the internal (polarization) charge density. This equation can easily be solved: $\mathbf{E}=-4 \pi \mathbf{P}=-4 \pi n q \mathbf{u}$ within this approximation (up to an irrelevant $c u r l$ ), so that the equation of motion (10) reads

$$
\left(\omega^{2}-\omega_{c}^{2}-\omega_{p}^{2}+i \omega \gamma\right) \mathbf{u}=-\frac{q}{m} \mathbf{E}_{0}
$$

this is the well-kown polarizability $\alpha(\omega)$,

$$
\mathbf{u}=-\frac{q}{m} \mathbf{E}_{0} \frac{1}{\omega^{2}-\omega_{c}^{2}-\omega_{p}^{2}+i \omega \gamma}=\frac{1}{n q} \alpha(\omega) \mathbf{E}_{0}
$$

we can see the presence of the plasmon eigenmode with frequency $\sqrt{\omega_{c}^{2}+\omega_{p}^{2}}$.

It is worth noting that for a point external charge $\operatorname{div} \mathbf{E}_{0}=4 \pi q_{0} \delta(\mathbf{r})$, the field is $\mathbf{E}_{0}=q_{0} \mathbf{r} / r^{3}$ and the displacement is given by

$$
\mathbf{P}=n q \mathbf{u}=\alpha(\omega) q_{0} \frac{\mathbf{r}}{r^{3}} ;
$$

the displacement acquires a maximum value near the external point charge and decreases like $1 / r^{2}$. We note that, in principle, knowing the displacement (polarization) for an external point charge we can get the displacement (polarization) for an external charge of any shape.

The result obtained above is valid for an infinite piece of matter. As it is well known, for finite-size bodies, the 
relationship between $\mathbf{P}$ and $\mathbf{E}$ is $\mathbf{E}=-4 \pi f \mathbf{P}$, where $f$ is the (de-) polarizing factor; the polarizability is modified according to

$$
\begin{gathered}
\alpha(\omega)=-\frac{\omega_{p}^{2}}{4 \pi} \frac{1}{\omega^{2}-\omega_{c}^{2}-\omega_{p}^{2}+i \omega \gamma} \rightarrow \\
\rightarrow \alpha(\omega)=-\frac{\omega_{p}^{2}}{4 \pi} \frac{1}{\omega^{2}-f \omega_{c}^{2}-\omega_{p}^{2}+i \omega \gamma} .
\end{gathered}
$$

Indeed, the presence of the surface of a finite-size body, as well as the boundary conditions (including, especially, the continuity conditions for the circular variables), lead to a charge density different from $\rho=-\operatorname{div} \mathbf{P}=-n q d i v \mathbf{u}$. For instance, for a semi-infinite body with a plane surface we get $f=1 / 2$, for the lowest mode of a sphere $f=1 / 3$, etc.

It is worth deriving explicitly the relationship between the polarizability $\alpha$ and the dielectric function $\varepsilon$; indeed, according to their definitions, we have $\mathbf{P}=\chi\left(\mathbf{E}_{0}+\mathbf{E}\right)=$ $\alpha \mathbf{E}_{0}$, where $\chi=(\varepsilon-1) / 4 \pi$ is the electric susceptibility; making use of $\mathbf{E}=-4 \pi f \mathbf{P}=-4 \pi f \alpha \mathbf{E}_{0}$, we get immediately $4 \pi \alpha=(\varepsilon-1) /(1-f+f \varepsilon)$. For sphere $f=1 / 3$ and this relationship is the well-known Clausius-Mossotti (or Lorenz-Lorentz) relation.[5]-[9],[17]

\section{Surface plasmons}

Let us suppose a half-space with a plane surface at $z=0$, extending over the region $z>0 .{ }^{1}$ Equation of motion (10) is preserved; it is convenient to write it as

$$
\left(\omega^{2}-\omega_{c}^{2}+i \omega \gamma\right)\left(\mathbf{u}, u_{z}\right)=-\frac{q}{m}\left(\mathbf{E}_{0}+\mathbf{E}\right), z>0
$$

where $\mathbf{u}$ is the in-plane displacement vector (parallel to the surface) and $u_{z}$ is the $z$-component of the displacement (perpendicular to the surface). The displacement field reads now $\left(\mathbf{u}, u_{z}\right) \theta(z)$, where $\theta(z)=1$ for $z>0$, $\theta(z)=0$ for $z<0$ is the step function; the charge density is $\rho=-n q \operatorname{div}\left[\left(\mathbf{u}, u_{z}\right) \theta(z)\right]$, which can be written as

$$
\begin{gathered}
\rho=-n q \operatorname{div}\left[\left(\mathbf{u}, u_{z}\right) \theta(z)\right]= \\
=-n q\left[\operatorname{div}\left(\mathbf{u}, u_{z}\right)\right] \theta(z)-n q u_{z}(z=0) \delta(z),
\end{gathered}
$$

where $\delta(z)$ is Dirac's delta function. We can see that there is a "volume" charge density $-n q\left[\operatorname{div}\left(\mathbf{u}, u_{z}\right)\right] \theta(z)$, which generates the "volume" field $\mathbf{E}_{v}=-4 \pi n q\left(\mathbf{u}, u_{z}\right) \theta(z)$ (equal to $-4 \pi \mathbf{P}$, where $\mathbf{P}=n q\left(\mathbf{u} . u_{z}\right) \theta(z)$ is the polarization) and there is an additional "surface" charge density $\rho_{s}=-n q u_{z}(z=0) \delta(z)$, corresponding to a surface charge density $n q u_{z}(z=0)$. The "volume" charge corresponds to "volume" plasmons, with frequency $\sqrt{\omega_{c}^{2}+\omega_{p}^{2}}$. For usual external fields the "volume" charge density does not buid up inside the body, so we may leave it aside. We

${ }^{1}$ The half-space does not fulfill rigourously the conditions of quasistatic fields, because of their infinite extension. are left with the "surface" charge density and the equation for the internal (polarization) electric field

$$
\operatorname{div} \mathbf{E}=4 \pi \rho_{s}=-4 \pi n q u_{z}(z=0) \delta(z) ;
$$

the equation of motion reads

$$
\left(\omega^{2}-\omega_{c}^{2}+i \omega \gamma\right)\left(\mathbf{u}, u_{z}\right)=-\frac{q}{m}\left(\mathbf{E}_{0}+\mathbf{E}_{s}\right), z>0 ;
$$

it remains to solve Gauss's equation (18) for the surface field.

This is a typical problem for finite-size bodies in the quasi-static approximation. Using $\mathbf{E}_{s}=-\operatorname{grad} \Phi_{s}$, it amounts to solving the Poisson equation

$$
\Delta \Phi_{s}=4 \pi n q u_{z}(z=0) \delta(z)
$$

for the surface term. Equation (20) is also the Green function's equation for the laplacian (with a $\delta$-source). ${ }^{2}$ The standard way of tackling such equations consists in using a complete expansion in orthogonal eigenfunctions corresponding to the free coordinates. In the present case this is a Fourier expansion along the $x, y$-coordinates, with the wavevector $\mathbf{k}$. Leaving aside the irrelevant arguments, we get

$$
\frac{d^{2} \Phi_{s}}{d z^{2}}-k^{2} \Phi_{s}=4 \pi n q u_{z}(z=0) \delta(z)
$$

with the solution

$$
\Phi_{s}=-\frac{2 \pi n q u_{z}(z=0)}{k} e^{-k|z|} ;
$$

we get the surface field

$$
\mathbf{E}_{s}=2 \pi n q\left(\frac{i \mathbf{k}}{k},-\operatorname{sgn}(z)\right) u_{z}(z=0) e^{-k|z|} .
$$

We denote the in-plane vectors by the subscript $\|$; the equation of motion (19) splits into two equations

$$
\begin{aligned}
& \left(\omega^{2}-\omega_{c}^{2}+i \omega \gamma\right) \mathbf{u}_{\|}=-\frac{q}{m} \mathbf{E}_{0 \|}- \\
& -\frac{i}{2} \omega_{p}^{2} u_{z}(z=0) \frac{\mathbf{k}}{k} e^{-k z}, z>0 \\
& \left(\omega^{2}-\omega_{c}^{2}+i \omega \gamma\right) u_{z}=-\frac{q}{m} E_{0 z}+ \\
& +\frac{1}{2} \omega_{p}^{2} u_{z}(z=0) e^{-k z}, z>0 .
\end{aligned}
$$

From the second equation we get

$$
u_{z}(z=0)=-\frac{q}{m} \frac{E_{0 z}(z=0)}{\omega^{2}-\omega_{c}^{2}-\frac{1}{2} \omega_{p}^{2}+i \omega \gamma}
$$

and

$$
\begin{gathered}
u_{z}=-\frac{q}{m} \frac{1}{\omega^{2}-\omega_{c}^{2}+i \omega \gamma} . \\
\cdot\left[E_{0 z}+\frac{\omega_{p}^{2}}{2} \frac{E_{0 z}(z=0)}{\omega^{2}-\omega_{c}^{2}-\frac{1}{2} \omega_{p}^{2}+i \omega \gamma} e^{-k z}\right] ;
\end{gathered}
$$

\footnotetext{
${ }^{2}$ In time-dependent problems equation (20) is replaced by the Helmholtz equation for the Green function, of the form $\Delta \Phi+$ $\left(\omega^{2} / c^{2}\right) \Phi=$ const $\cdot \delta(\mathbf{r})$.
} 
while from the first equation (24) we get

$$
\begin{gathered}
\mathbf{u}_{\|}=-\frac{q}{m} \frac{1}{\omega^{2}-\omega_{c}^{2}+i \omega \gamma} \\
\cdot\left[\mathbf{E}_{0 \|}-i \frac{\omega_{p}^{2}}{2} \frac{E_{0 z}(z=0)}{\omega^{2}-\omega_{c}^{2}-\frac{1}{2} \omega_{p}^{2}+i \omega \gamma} \frac{\mathbf{k}}{k} e^{-k z}\right] .
\end{gathered}
$$

It is convenient to introduce the "surface" polarizability

$$
\alpha_{s}(\omega)=-\frac{\omega_{p}^{2}}{8 \pi} \frac{1}{\omega^{2}-\omega_{c}^{2}-\frac{1}{2} \omega_{p}^{2}+i \omega \gamma}
$$

beside the "volume" polarizability

$$
\alpha(\omega)=-\frac{\omega_{p}^{2}}{4 \pi} \frac{1}{\omega^{2}-\omega_{p}^{2}-\omega_{c}^{2}+i \omega \gamma}
$$

(and recall the susceptibility

$$
\left.\chi(\omega)=-\frac{\omega_{p}^{2}}{4 \pi} \frac{1}{\omega^{2}-\omega_{c}^{2}}\right)
$$

the displacements can then be written as

$$
\begin{gathered}
u_{z}=\frac{1}{n q} \chi(\omega) \cdot \\
\cdot\left[E_{0 z}-4 \pi \alpha_{s}(\omega) E_{0 z}(z=0) e^{-k z}\right], \\
\mathbf{u}_{\|}=\frac{1}{n q} \chi(\omega) \cdot \\
\cdot\left[\mathbf{E}_{0 \|}+4 \pi i \alpha_{s}(\omega) E_{0 z}(z=0) \frac{\mathbf{k}}{k} e^{-k z}\right] .
\end{gathered}
$$

First, we note the occurrence of an additonal, surface eigenmode with frequency $\sqrt{\omega_{c}^{2}+\omega_{p}^{2} / 2}$; it is the surface plasmon. Next, we note the polarization $\mathbf{P}=n q\left(\mathbf{u}, u_{z}\right)$ (for $z>0)$ and check the relationship $\mathbf{P}=\chi\left(\mathbf{E}_{s}+\mathbf{E}_{0}\right)$, by using equations (23), (26) and (27), as well as the continuity at the surface of the tangential components of the internal (polarization) electric field $\mathbf{E}_{s}$ and the transverse components of the electric displacement $\mathbf{D}=\mathbf{E}_{s}+4 \pi \mathbf{P}$. In addition for usual quasi-static external fields we have the conditions $\operatorname{div} \mathbf{E}_{0}=0$ and $\operatorname{curl} \mathbf{E}_{0}=0$, which lead, for Fourier transforms, to $\mathbf{E}_{0}=\mathbf{E}_{0}(z=0) e^{-k z}$, as well as to $u_{1}=-i u_{z}$. Consequently, from equations (26) and (27), we can also write the response as

$$
\left(\mathbf{u}, u_{z}\right)=-\frac{q}{m} \frac{1}{\omega^{2}-\omega_{c}^{2}-\frac{1}{2} \omega_{p}^{2}+i \omega \gamma} \mathbf{E}_{0}
$$

\section{as expected.}

By using the method described here we can treat a serie of interesting situations within the quasi-static approximation, like a point charge or a point dipole placed in the vicinity of a half-space, or any other external field acting upon a half-space, or two half-spaces separated by a gap, a slab, etc. In all these situations we expect a resonance arising from the surface plasmons.

\section{Half-space}

The half-space can be given a full electromagnetic solution.[10, 11],[13] For a semi-infinite (homogeneous) body (half-space) extending over the region $z>d$ we take the polarization as

$$
\mathbf{P}=n q\left(\mathbf{u}, u_{z}\right) \theta(z-d)
$$

where $\theta(z)=0$ for $z<0$ and $\theta(z)=1$ for $z>0$ is the step function. The polarization charge and current densities are given by

$$
\begin{gathered}
\rho=-n q\left(\operatorname{div} \mathbf{u}+\frac{\partial u_{z}}{\partial z}\right) \theta(z-d)-n q u_{z}(d) \delta(z-d), \\
\mathbf{j}=n q\left(\dot{\mathbf{u}}, \dot{u}_{z}\right) \theta(z-d) .
\end{gathered}
$$

We use Fourier decompositions of the type

$$
\mathbf{u}(\mathbf{r}, z ; t)=\frac{1}{2 \pi} \sum_{\mathbf{k}} \int d \omega \mathbf{u}(\mathbf{k}, z ; \omega) e^{-i \omega t+i \mathbf{k r}}
$$

where $\mathbf{R}=(\mathbf{r}, z)$, and may omit ocassionally the arguments $\mathbf{k}, \omega$, writing simply $\mathbf{u}(z)$, or $\mathbf{u}$. The electromagnetic potentials given by

$$
\begin{aligned}
& \Phi(\mathbf{R}, t)=\int d \mathbf{R}^{\prime} \frac{\rho\left(\mathbf{R}^{\prime}, t-\left|\mathbf{R}-\mathbf{R}^{\prime}\right| / c\right)}{\left|\mathbf{R}-\mathbf{R}^{\prime}\right|} \\
& \mathbf{A}(\mathbf{R}, t)=\frac{1}{c} \int d \mathbf{R}^{\prime} \frac{\mathbf{j}\left(\mathbf{R}^{\prime}, t-\left|\mathbf{R}-\mathbf{R}^{\prime}\right| / c\right)}{\left|\mathbf{R}-\mathbf{R}^{\prime}\right|}
\end{aligned}
$$

include the "retarded" Coulomb potential $e^{i \frac{\omega}{c}\left|\mathbf{R}-\mathbf{R}^{\prime}\right|} /\left|\mathbf{R}-\mathbf{R}^{\prime}\right|$, for which we use the well-known decomposition

$$
\frac{e^{i \lambda\left|\mathbf{R}-\mathbf{R}^{\prime}\right|}}{\left|\mathbf{R}-\mathbf{R}^{\prime}\right|}=\frac{i}{2 \pi} \int d \mathbf{k} \frac{1}{\kappa} e^{i \mathbf{k}\left(\mathbf{r}-\mathbf{r}^{\prime}\right)} e^{i \kappa\left|z-z^{\prime}\right|},
$$

where $\lambda=\omega / c$ and $\kappa=\sqrt{\lambda^{2}-k^{2}}$. It is more convenient to compute first the vector potential $\mathbf{A}$ and then derive the scalar potential $\Phi$ from the gauge equation $\operatorname{div} \mathbf{A}-i \lambda \Phi=$ 0 . The calculations are straightforward and we get the Fourier tranforms of the potentials

$$
\begin{gathered}
\Phi(\mathbf{k}, z ; \omega)==\frac{2 \pi}{\kappa} \int_{d}^{\infty} d z^{\prime} \mathbf{k} \mathbf{u} e^{i \kappa\left|z-z^{\prime}\right|}- \\
-\frac{2 \pi i}{\kappa} \frac{\partial}{\partial z} \int_{d}^{\infty} d z^{\prime} u_{z} e^{i \kappa\left|z-z^{\prime}\right|} \\
\mathbf{A}(\mathbf{k}, z ; \omega)=\frac{2 \pi \lambda}{\kappa} \int_{d}^{\infty} d z^{\prime}\left(\mathbf{u}, u_{z}\right) e^{i \kappa\left|z-z^{\prime}\right|}
\end{gathered}
$$

(where we have left aside the factor $n q$; it is restored in the final formulae). In order to compute the electric field $(\mathbf{E}=i \lambda \mathbf{A}-\operatorname{grad} \Phi)$ it is convenient to refer the in-plane vectors (i.e., vectors parallel with the surface of the halfspace) to the vectors $\mathbf{k}$ and $\mathbf{k}_{\perp}=e_{z} \times \mathbf{k}$, where $e_{z}$ is the unit vector along the $z$-direction; for instance, we write

$$
\mathbf{u}=u_{1} \frac{\mathbf{k}}{k}+u_{2} \frac{\mathbf{k}_{\perp}}{k}
$$


and a similar representation for the electric field parallel with the surface of the half-space. We get the electric field

$$
\begin{gathered}
E_{1}=2 \pi i \kappa \int_{d}^{\infty} d z^{\prime} u_{1} e^{i \kappa\left|z-z^{\prime}\right|} \\
-\frac{2 \pi k}{\kappa} \frac{\partial}{\partial z} \int_{d}^{\infty} d z^{\prime} u_{z} e^{i \kappa\left|z-z^{\prime}\right|}, \\
E_{2}=\frac{2 \pi i \lambda^{2}}{\kappa} \int_{d}^{\infty} d z^{\prime} u_{2} e^{i \kappa\left|z-z^{\prime}\right|}, \\
E_{z}=-\frac{2 \pi k}{\kappa} \frac{\partial}{\partial z} \int_{d}^{\infty} d z^{\prime} u_{1} e^{i \kappa\left|z-z^{\prime}\right|}+ \\
+\frac{2 \pi i k^{2}}{\kappa} \int_{d}^{\infty} d z^{\prime} u_{z} e^{i \kappa\left|z-z^{\prime}\right|}-4 \pi u_{z} \theta(z-d) .
\end{gathered}
$$

Making use of equations (40), we can check easily the equalities

$$
\begin{gathered}
i k E_{1}+\frac{\partial E_{z}}{\partial z}=-4 \pi\left(i k u_{1}+\frac{\partial u_{z}}{\partial z}\right) \theta(z-d) \\
-4 \pi u_{z}(z=d) \delta(z-d)
\end{gathered}
$$

which is an expression of Gauss's law, and

$$
k \frac{\partial E_{1}}{\partial z}+i \kappa^{2} E_{z}=-4 \pi i \lambda^{2} u_{z} \theta(z-d),
$$

which reflects Faraday's and Maxwell-Ampere's equations. From equation (41), we can check the transversality condition $\operatorname{div} \mathbf{E}=0$ for the electric field outside the half-space $(z<d)$.

We use now the equation of motion (2) (with $\gamma=0$ ) for $E_{2}$ given by equation (40) and for the combinations $i k u_{1}+$ $\partial u_{z} / \partial z$ and $k \partial u_{1} / \partial z+i \kappa^{2} u_{z}$ in the region $z>d$. Taking into account that $d i v \mathbf{E}_{0}=0$ and $k \partial E_{01} / \partial z+i \kappa^{2} E_{0 z}=0$ (for a plane wave) we get

$$
i k u_{1}+\frac{\partial u_{z}}{\partial z}=0, k \frac{\partial u_{1}}{\partial z}+i \kappa^{\prime 2} u_{z}=0
$$

or

$$
\frac{\partial^{2} \mathbf{u}}{\partial z^{2}}+\kappa^{\prime 2} \mathbf{u}=0
$$

where

$$
\kappa^{\prime 2}=\kappa^{2}-\frac{\lambda^{2} \omega_{p}^{2}}{\omega^{2}-\omega_{c}^{2}} .
$$

We emphasize that the rhs of equation (44) is vanishing only for a transverse external field. The components $u_{1,2}$ of the displacement field are given by $u_{1,2}=A_{1,2} e^{i \kappa^{\prime} z}$, where $A_{1,2}$ are constants, while $u_{z}=-\left(k / \kappa^{\prime}\right) A_{1} e^{i \kappa^{\prime} z}$ (we restrict ourselves to outgoing waves, $\kappa^{\prime}>0$ ). The total electric field inside the half-space is given by the equation of motion (2):

$$
\mathbf{E}_{t}=-\frac{m}{q}\left(\omega^{2}-\omega_{c}^{2}\right) \mathbf{u}
$$

for $z>d$. We can see that the field propagates in the half-space with a modified wavevector $\kappa^{\prime}$, according to the Ewald-Oseen extinction theorem.[18]-[20] The modified wavevector $\kappa^{\prime}$ given by equation (45) can also be written as

$$
\kappa^{\prime 2}=\varepsilon \frac{\omega^{2}}{c^{2}}-k^{2},
$$

where

$$
\varepsilon=1-\frac{\omega_{p}^{2}}{\omega^{2}-\omega_{c}^{2}}
$$

is the dielectric function (as given by equation (16), for instance). We can check the well-known polaritonic dispersion relation $\varepsilon \omega^{2}=c^{2} K^{\prime 2}$, where $\mathbf{K}^{\prime}=\left(\mathbf{k}, \kappa^{\prime}\right)$ is the wavevector.

The amplitudes $A_{1,2}$ can be derived from the original equation (2) and the field equations (40) (for $z>d$ ). We get

$$
\begin{aligned}
& \frac{1}{2} A_{1} \omega_{p}^{2} \frac{\kappa \kappa^{\prime}+k^{2}}{\kappa^{\prime}\left(\kappa^{\prime}-\kappa\right)} e^{i\left(\kappa^{\prime}-\kappa\right) d} e^{i \kappa z}=\frac{q}{m} E_{01}, \\
& \frac{1}{2} A_{2} \omega_{p}^{2} \frac{\lambda^{2}}{\kappa\left(\kappa^{\prime}-\kappa\right)} e^{i\left(\kappa^{\prime}-\kappa\right) d} e^{i \kappa z}=\frac{q}{m} E_{02} .
\end{aligned}
$$

The (polarization) electric field, both inside and outside the half-space, can be computed from equations (40). We get

$$
\begin{gathered}
E_{1}=-4 \pi n q A_{1} \frac{\omega^{2}-\omega_{c}^{2}}{\omega_{p}^{2}} e^{i \kappa^{\prime} z}- \\
-2 \pi n q A_{1} \frac{\kappa \kappa^{\prime}+k^{2}}{\kappa^{\prime}\left(\kappa^{\prime}-\kappa\right)} e^{i\left(\kappa^{\prime}-\kappa\right) d} e^{i \kappa z}, z>d \\
E_{2}=-4 \pi n q A_{2} \frac{\omega^{2}-\omega_{c}^{2}}{\omega_{p}^{2}} e^{i \kappa^{\prime} z}- \\
-2 \pi n q A_{2} \frac{\lambda^{2}}{\kappa\left(\kappa^{\prime}-\kappa\right)} e^{i\left(\kappa^{\prime}-\kappa\right) d} e^{i \kappa z}, z>d, \\
E_{z}=4 \pi n q A_{1} \frac{k\left(\omega^{2}-\omega_{c}^{2}\right)}{\kappa^{\prime} \omega_{p}^{2}} e^{i \kappa^{\prime} z}+ \\
+2 \pi n q A_{1} \frac{k\left(\kappa \kappa^{\prime}+k^{2}\right)}{\kappa \kappa^{\prime}\left(\kappa^{\prime}-\kappa\right)} e^{i\left(\kappa^{\prime}-\kappa\right) d} e^{i \kappa z}, z>d .
\end{gathered}
$$

for $z>d$. It is worth noting that the polarization electric field, as given by equations (50), includes both the external field $\sim e^{i \kappa z}$ (with opposite sign) and the displacement field $\mathbf{u} \sim e^{i \kappa^{\prime} z}$. This can be checked easily by using equations (49) and (50). The (polarization) electric field outside the half-space (in the region $z<d$ ) is given by

$$
\begin{aligned}
& E_{1}=-2 \pi n q A_{1} \frac{\kappa \kappa^{\prime}-k^{2}}{\kappa^{\prime}\left(\kappa^{\prime}+\kappa\right)} . \\
& \cdot e^{i\left(\kappa^{\prime}+\kappa\right) d} e^{-i \kappa z}, z<d, \\
& E_{2}=-2 \pi n q A_{2} \frac{\lambda^{2}}{\kappa\left(\kappa^{\prime}+\kappa\right)} . \\
& \cdot e^{i\left(\kappa^{\prime}+\kappa\right) d} e^{-i \kappa z}, z<d
\end{aligned}
$$

and $E_{z}=(k / \kappa) E_{1}$ for $z<d$. We can see that it is the field reflected by the half-space $(\kappa \rightarrow-\kappa)$. Making use of equations (49) and (51) we get also the total electric field $\mathbf{E}_{t}=\mathbf{E}+\mathbf{E}_{0}$ outside the half-space and the magnetic field $\mathbf{H}=$ curl $\mathbf{A}$ both inside and outside the half-space. The amplitudes $A_{1,2}$ can be viewed either as being determined by the external field $\mathbf{E}_{0}$ (and $\mathbf{H}_{0}$ ) through equations (49), or as free parameters. In the latter case equations (49) are not valid anymore, but the (polarization) electric and magnetic fields (given, for instance, by equations (50), (51)) 
hold. We can check also that all the fields are continuous at the surface $z=d$, except for $E_{z}$ and $E_{t z}$, which exhibit a discontinuity $\left(E_{t z}\left(z=d^{-}\right)=\varepsilon E_{t z}\left(z=d^{+}\right)\right.$), as expected. In particular, the surface plasmon-polariton mode given by equation (6) is obtained from the vanishing denominator $\kappa \kappa^{\prime}+k^{2}$ of the response (equations (49)) for both $\kappa$ and $\kappa^{\prime}$ purely imaginary, which means that both the incident and the refracted waves are localized at the surface and propagates only along the surface.

Having known the electromagnetic solution for a halfspace we can derive the Fresnel formulae for reflection and refraction coefficients.[10, 11] Similarly, we can treat two half-spaces, a slab (or a two dimensional sheet). In particular we can derive the van der Waals-London-Casimir force for such electromagnetically coupled bodies,[13] or we can treat other interesting situations, like the presence of the inhomogeneities, rough surfaces,[16] etc. Here, we present a very interesting situation concerning a point-dipole and a half-space.

\section{Point dipole and half-space}

A point dipole placed at the origin has a charge density and a current density given by

$$
\begin{gathered}
\rho=-n_{0} q v\left[\left(\mathbf{w}, w_{z}\right) \operatorname{grad}\right] \delta(\mathbf{R}), \\
\mathbf{j}=n_{0} q v\left(\dot{\mathbf{w}}, \dot{w}_{z}\right) \delta(\mathbf{R})
\end{gathered}
$$

where $n_{0}$ is its density of charges $q$ (with mass $m$ ), $v$ is the (volume) of the dipole, $\mathbf{w}$ is the displacement field in the $x, y$-plane and $w_{z}$ is the $z$-component of the displacement field; the displacement field depends only on the time. These charge and current densities introduced in equations (36) give the electromagnetic potentials; the vector potential is

$$
\mathbf{A}_{0}=-i \lambda n_{0} q v\left(\mathbf{w}, w_{z}\right) \frac{e^{i \lambda R}}{R}
$$

(temporal Fourier transform), where $\lambda=\omega / c$; the scalar potential is conveniently obtained from the Lorenz gauge $\operatorname{div} \mathbf{A}_{0}-i \lambda \Phi_{0}=0$ :

$$
\Phi_{0}=-n_{0} q v\left[\left(\mathbf{w}, w_{z}\right) \operatorname{grad}\right] \frac{e^{i \lambda R}}{R} .
$$

The electric field can be calculated from $\mathbf{E}_{0}=i \lambda \mathbf{A}_{0}-$ $\operatorname{grad} \Phi_{0}$. We represent the spherical wave by

$$
\frac{e^{i \lambda R}}{R}=\frac{i}{2 \pi} \int d \mathbf{k} \frac{1}{\kappa} e^{i \mathbf{k r}} e^{i \kappa|z|}
$$

where $\mathbf{R}=(\mathbf{r}, z)$ and $\kappa=\sqrt{\lambda^{2}-k^{2}}$; then, we select the k-component by using $\sum_{\mathbf{k}}=\left[A /(2 \pi)^{2}\right] \int d \mathbf{k}$, where $A$ is the in-plane $((x, y)$-plane) area; it is convenient to use the projections of the electric field on $\mathbf{k}\left(E_{01}\right)$ and $\mathbf{k}_{\perp}\left(E_{02}\right)$, where $\mathbf{k}_{\perp}$ is perpendicular to $\mathbf{k}$ and has the same magnitude $k$. We get

$$
\begin{gathered}
E_{01}=\frac{2 \pi i n_{0} q v}{A}\left[\kappa w_{1}-k w_{z} \operatorname{sgn}(z)\right] e^{i \kappa|z|}, \\
E_{02}=\frac{2 \pi i n_{0} q v}{A} \cdot \frac{\lambda^{2}}{\kappa} w_{2} e^{i \kappa|z|}, \\
E_{0 z}=\frac{2 \pi n_{0} q v}{A} . \\
{\left[\frac{i k^{2}}{\kappa} w_{z}-i k w_{1} \operatorname{sgn}(z)-2 w_{z} \delta(z)\right] e^{i \kappa|z|} ;}
\end{gathered}
$$

we can check that this field is transverse $\left(\operatorname{div} \mathbf{E}_{0}=0\right)$.

From the calculations for a half-space described before we know the displacement field $u_{1,2}=A_{1,2} e^{i \kappa^{\prime} z}, u_{z}=$ $-\left(k / \kappa^{\prime}\right) A_{1} e^{i \kappa^{\prime} z}$, for $z>d$, where

$$
\kappa^{\prime}=\sqrt{\kappa^{2}-\frac{\lambda^{2} \omega_{p}^{2}}{\omega^{2}-\omega_{c}^{2}}}=\sqrt{\lambda^{2} \varepsilon-k^{2}}
$$

and the coefficients $A_{1,2}$ are given by

$$
\begin{gathered}
\frac{1}{2} A_{1} \omega_{p}^{2} \frac{\kappa \kappa^{\prime}+k^{2}}{\kappa^{\prime}\left(\kappa^{\prime}-\kappa\right)} e^{i\left(\kappa^{\prime}-\kappa\right) d} e^{i \kappa z}= \\
=\frac{q}{m} E_{01}, \\
\frac{1}{2} A_{2} \omega_{p}^{2} \frac{\lambda^{2}}{\kappa\left(\kappa^{\prime}-\kappa\right)} e^{i\left(\kappa^{\prime}-\kappa\right) d} e^{i \kappa z}= \\
=\frac{q}{m} E_{02},
\end{gathered}
$$

$\omega_{p}$ being the plasma frequency for the half-space (equations (49)). The field reflected by the half-space (equations (51)) is given by

$$
\begin{gathered}
E_{r 1}=-2 \pi n q A_{1} \frac{\kappa \kappa^{\prime}-k^{2}}{\kappa^{\prime}\left(\kappa^{\prime}+\kappa\right)} . \\
\cdot e^{i\left(\kappa^{\prime}+\kappa\right) d} e^{-i \kappa z}, z<d \\
E_{r 2}=-2 \pi n q A_{2} \frac{\lambda^{2}}{\kappa\left(\kappa^{\prime}+\kappa\right)} . \\
\cdot e^{i\left(\kappa^{\prime}+\kappa\right) d} e^{-i \kappa z}, z<d
\end{gathered}
$$

and $E_{r z}=(k / \kappa) E_{1}$ for $z<d$, where $n$ is the density of charges in the half-space. By using equations (58) we can express this reflected field by means of the field $\mathbf{E}_{0}$ and, by using equations (56), we can express it in terms of the displacement $\mathbf{W}=\left(\mathbf{w}, w_{z}\right)$. The reflected field plays the role of an external field in the equation of motion of the dipole displacement field[21]

$$
\left(\omega^{2}-\omega_{0}^{2}\right) \mathbf{W}=-\frac{q}{m} \mathbf{E}_{r}(\mathbf{r}=0, z=0 ; \omega),
$$

where $\omega_{0}$ is a characteristic frequency of the point dipole; we get

$$
\begin{gathered}
\left(\omega^{2}-\omega_{0}^{2}\right) \mathbf{W}=\frac{i \omega_{p 0}^{2} v}{A} . \\
\sum_{\mathbf{k}}\left\{\frac{\left(\kappa^{\prime}-\kappa\right)\left(\kappa \kappa^{\prime}-k^{2}\right)}{\left(\kappa^{\prime}+\kappa\right)\left(\kappa \kappa^{\prime}+k^{2}\right)}\left(\kappa w_{1}-k w_{z}\right)\left(\frac{\mathbf{k}}{k}+\frac{k}{\kappa} \mathbf{e}_{z}\right)+\right. \\
\left.+\frac{\lambda^{2}\left(\kappa^{\prime}-\kappa\right)}{\kappa\left(\kappa^{\prime}+\kappa\right)} w_{2} \frac{\mathbf{k}_{\perp}}{k}\right\} e^{2 i \kappa d}
\end{gathered}
$$


where $\omega_{p 0}=\sqrt{4 \pi n_{0} q^{2} / m}$. Here we use $\mathbf{k}=$ $k(\cos \varphi, \sin \varphi)$ and $\mathbf{k}_{\perp}=k(-\sin \varphi, \cos \varphi)$ and integrate over the angle $\varphi$. We get the equations

$$
\begin{gathered}
\left(\omega^{2}-\omega_{0}^{2}\right) w=\frac{i \omega_{p 0}^{2} v}{8 \pi} . \\
\cdot \int d k \cdot k\left[\frac{\kappa\left(\kappa^{\prime}-\kappa\right)\left(\kappa \kappa^{\prime}-k^{2}\right)}{\left(\kappa^{\prime}+\kappa\right)\left(\kappa \kappa^{\prime}+k^{2}\right)}+\frac{\lambda^{2}\left(\kappa^{\prime}-\kappa\right)}{\kappa\left(\kappa^{\prime}+\kappa\right)}\right] w e^{2 i \kappa d} \\
\left(\omega^{2}-\omega_{0}^{2}\right) w_{z}=-\frac{i \omega_{p 0}^{2} v}{4 \pi} . \\
\cdot \int d k \cdot k^{3} \frac{\left(\kappa^{\prime}-\kappa\right)\left(\kappa \kappa^{\prime}-k^{2}\right)}{\kappa\left(\kappa^{\prime}+\kappa\right)\left(\kappa \kappa^{\prime}+k^{2}\right)} w_{z} e^{2 i \kappa d}
\end{gathered}
$$

$w$ is an in-plane component of the displacement, the same equation holds for the other component. These equations can also be written as

$$
\begin{gathered}
\left(\omega^{2}-\omega_{0}^{2}\right) w=\frac{i \omega_{p 0}^{2} v}{8 \pi} . \\
\cdot \int d k \cdot k\left[\frac{\kappa\left(\kappa \varepsilon-\kappa^{\prime}\right)}{\kappa \varepsilon+\kappa^{\prime}}+\frac{\lambda^{2}\left(\kappa^{\prime}-\kappa\right)}{\kappa\left(\kappa^{\prime}+\kappa\right)}\right] w e^{2 i \kappa d}, \\
\left(\omega^{2}-\omega_{0}^{2}\right) w_{z}=-\frac{i \omega_{p 0}^{2} v}{4 \pi} . \\
\cdot \int d k \cdot k^{3} \frac{\kappa \varepsilon-\kappa^{\prime}}{\kappa\left(\kappa \varepsilon+\kappa^{\prime}\right)} w_{z} e^{2 i \kappa d}
\end{gathered}
$$

it is easy to see that these equations give the eigenfrequencies of the coupled point dipole and half-space.

We notice first that the dipole volume $v$ should be much smaller than any relevant scale; this means that the solution $\omega$ should lie near $\omega_{0}$ and the rhs of equations (63) should be treated as a small perturbation. Next, we notice that for large distances $d$ the main contribution to the integrals come from small values of $\kappa$, i.e. from $k$ near $\lambda$; this implies $d \lambda_{0}=d \omega_{0} / c \gg 1$ and small frequencies $\omega_{0}$ in comparison with $\omega_{c}, \omega_{p}$; the main contribution comes in this case from small values of $k$; this is the retarded case; it is easy to see that equations (63) have not solutions in this case: the waves are damped, except for some discrete values of the distance $d$; in general, the two bodies do not couple to each other in this case. For small distances $d\left(d \omega_{0} / c \ll 1\right)$, the main contribution to the integrals comes from large values of $k$, such as $\kappa \simeq \kappa^{\prime} \simeq i k$; the first equation (63) can be written approximately

$$
\omega^{2}-\omega_{0}^{2} \simeq \frac{i \omega_{p 0}^{2} v}{8 \pi} \frac{\varepsilon-1}{\varepsilon+1} \int d k \cdot i k^{2} e^{-2 k d} ;
$$

this is the non-retarded case. We get easily the solution

$$
\Omega_{\|} \simeq \omega_{0}-\frac{\omega_{p 0}^{2} v}{64 \pi \omega_{0}} \frac{\varepsilon_{0}-1}{\varepsilon_{0}+1} \cdot \frac{1}{d^{3}},
$$

where $\varepsilon_{0}=\varepsilon\left(\omega_{0}\right)$; hence, the zero-point energy $\hbar \Omega_{\|} / 2$ leads to the van der Waals-London force (for a point dipole coupled to a half-space); we should take into account the multiplicity 2 for the transverse oscillations and the contribution of the $z$-component, whose eigenfrequency is given by

$$
\Omega_{z} \simeq \omega_{0}-\frac{\omega_{p 0}^{2} v}{32 \pi \omega_{0}} \frac{\varepsilon_{0}-1}{\varepsilon_{0}+1} \cdot \frac{1}{d^{3}} .
$$

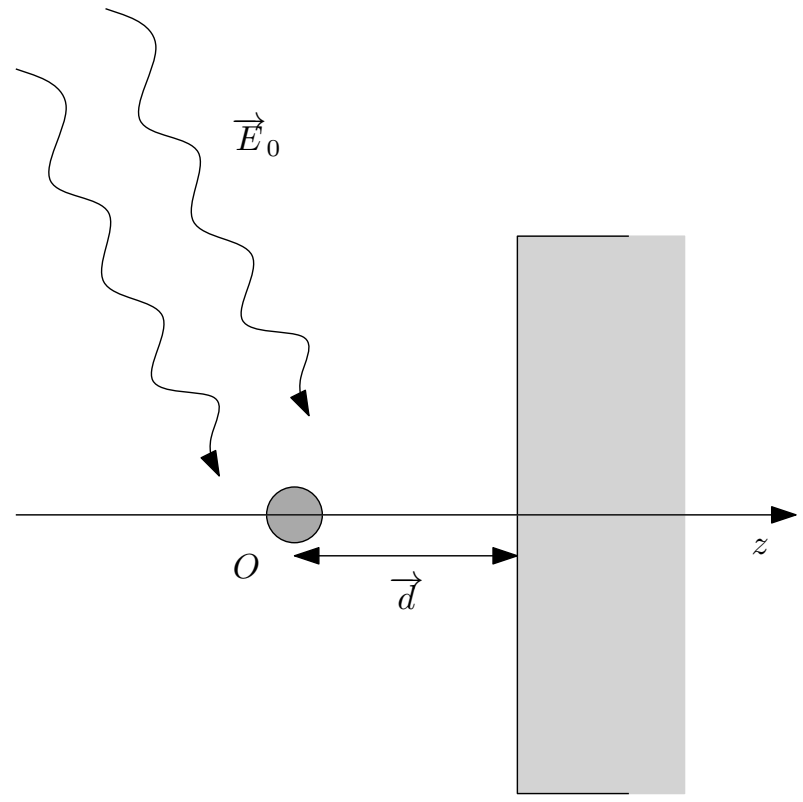

Figure 1: External field, point dipole and a half-space

Finally, we get the force

$$
F=-\frac{3 \hbar \omega_{p 0}^{2} v}{32 \pi \omega_{0}} \frac{\varepsilon_{0}-1}{\varepsilon_{0}+1} \cdot \frac{1}{d^{4}}
$$

Making use of $\varepsilon=1-\omega_{p}^{2} /\left(\omega^{2}-\omega_{c}^{2}\right)$, we note that one obtains an appreciable enhancement of this force for the characteristic frequency $\omega_{0}$ of the point dipole approaching the frequency $\sqrt{\omega_{c}^{2}+\omega_{p}^{2} / 2}$ of the surface plasmon.

\section{Response of a point dipole and a half-space}

We consider a point dipole induced by an external field, placed in the vicinity of a half-space. The equation of motion (60) acquires now an additional term, arising from the contribution of the external field. We take a monochromatic plane wave of the form

$$
\mathbf{E}_{0}(t, \mathbf{r}, z)=\mathbf{E}_{0} e^{-i \Omega t} e^{i\left(\mathbf{K r}+K_{z} z\right)}
$$

as an external (radiation) field. The calculations described above can easily be repeated in this case. We limit ourselves here to give the result (in the reference frame formed by the 
vectors $\mathbf{K}, \mathbf{K}_{\perp}$ and $\mathbf{K}_{z}$ :

$$
\begin{gathered}
\left(\omega^{2}-\omega_{0}^{2}\right) w_{1}=\frac{i \omega_{p 0}^{2} v}{8 \pi} w_{1} \cdot \\
\cdot \int d k \cdot k\left[\kappa \frac{\varepsilon \kappa-\kappa^{\prime}}{\varepsilon \kappa+\kappa^{\prime}}+\frac{\lambda^{2}\left(\kappa^{\prime}-\kappa\right)}{\kappa\left(\kappa^{\prime}+\kappa\right)}\right] e^{2 i \kappa d}+ \\
+\frac{2 \pi q}{m}\left[\frac{\varepsilon K_{z}-K_{z}^{\prime}}{\varepsilon K_{z}+K_{z}^{\prime}} e^{2 i K_{z} d}-1\right] E_{01} \delta(\omega-\Omega) \\
\left(\omega^{2}-\omega_{0}^{2}\right) w_{2}=\frac{i \omega_{p 0}^{2} v}{8 \pi} w_{2} \cdot \\
\cdot \int d k \cdot k\left[\kappa \frac{\varepsilon \kappa-\kappa^{\prime}}{\varepsilon \kappa+\kappa^{\prime}}+\frac{\lambda^{2}\left(\kappa^{\prime}-\kappa\right)}{\kappa\left(\kappa^{\prime}+\kappa\right)}\right] e^{2 i \kappa d}+ \\
+\frac{2 \pi q}{m}\left[\frac{K_{z}^{\prime}-K_{z}}{K_{z}^{\prime}+K_{z}} e^{2 i K_{z} d}-1\right] E_{02} \delta(\omega-\Omega) \\
\left(\omega^{2}-\omega_{0}^{2}\right) w_{z}=-\frac{i \omega_{p 0}^{2} v}{4 \pi} w_{z} \int d k \cdot k^{3} \frac{\varepsilon \kappa-\kappa^{\prime}}{\kappa\left(\varepsilon \kappa+\kappa^{\prime}\right)} e^{2 i \kappa d}- \\
-\frac{2 \pi q}{m}\left[\frac{\varepsilon K_{z}-K_{z}^{\prime}}{\varepsilon K_{z}+K_{z}^{\prime}} e^{2 i K_{z} d}+1\right] E_{0 z} \delta(\omega-\Omega)
\end{gathered}
$$

where $K_{z}^{\prime}=\sqrt{\varepsilon \Omega^{2} / c^{2}-K^{2}}$. As before, the main contributions to the integrals in equations (69) comes from large values of $k$; in addition, we are interested in small distances, such as $e^{2 i K_{z} d} \simeq 1$. We get easily

$$
\begin{gathered}
\left(\omega^{2}-\Omega_{\|}^{2}\right) w_{1}=-\frac{\cos r}{\sqrt{\varepsilon} \cos i+\cos r} . \\
\cdot \frac{4 \pi q}{m} E_{01} \delta(\omega-\Omega), \\
\left(\omega^{2}-\Omega_{\|}^{2}\right) w_{2}=-\frac{\cos i}{\cos i+\sqrt{\varepsilon} \cos r} . \\
\cdot \frac{4 \pi q}{m} E_{02} \delta(\omega-\Omega), \\
\left(\omega^{2}-\Omega_{z}^{2}\right) w_{z}=-\frac{\sqrt{\varepsilon} \cos i}{\sqrt{\varepsilon} \cos i+\cos r} . \\
\cdot \frac{4 \pi q}{m} E_{0 z} \delta(\omega-\Omega),
\end{gathered}
$$

where $i$ is the incidence angle, $r$ is the refraction angle ( $\sin r / \sin i=1 / \sqrt{\varepsilon}$ ) and $\Omega_{\|}, \Omega_{z}$ are given by equations (65) and (66).

The displacement field $w_{1,2, z}$ given by equations (70) represents the response of the induced point dipole placed in the vicinity of a half-space to an external radiation field; multiplied by $n_{0} q v$ it gives the induced dipole moment. Equations (70) answer the question regarding the effect of a semi-infinite solid placed in the vicinity of a small, neutral nano-particle which can acquire an induced dipole moment under the action of a radiation field. This is the typical problem in the surface enhanced Raman scattering.[22]-[26] We can see that the induced dipole may be enhanced appreciably for frequencies close to the resonance frequencies $\Omega_{\|, z}$. We can compare this effect with the absence of the semiinfinite solid, which is obtained by putting $\varepsilon=1$ (in this case $\Omega_{\|, z}$ reduce to $\omega_{0}$ ).

\section{Discussion}

First, in the analysis made above we have considered a point dipole, i.e. a dipole whose dimensions are much smaller in comparison with both distances of interest and the radiation wavelength. A molecule or a nano-particle may fulfill such requirements. Second, we consider an external electromagnetic radiation field (usually a plane wave) which acts upon a molecule or a nano-particle. In these circumstances, there occur optical transitions, driven by matrix elements of the electric dipole, such that the dipole is in fact an induced dipole. Usually, these transitions are coupled with vibration and rotation degrees of freedom, leading to a dipole radiation which exhibits additional frequencies beside the main, driving frequency of the external field. This is the well-known Raman effect.[27, 28] For large molecules or nanoparticles (but sufficiently small for being treated as point dipoles) the transitions imply the classical motion of the particle density, i.e. the classical motion of the electric polarization; therefore, a classical model for Raman scattering is justified and often employed. It is based on classical equations of motion of the displacement field $\mathbf{W}$, like equation (60), where the characteristic frequencies $\omega_{0}$ are the eigenfrequencies of the coupled polarization-rotation or polarization-vibration motion, which are effective in Raman scattering.

Now, let us assume that such a dipole is placed in the vicinity of a polarizable half-space (semi-infinite solid), as shown in Fig. 1. In this case, there happen two distinct things. First, the external radiation field (the electric field $\mathbf{E}_{0}$ given by equation (68)) gets reflected and refracted, according to the Fresnel formulae, as given above in equations (49)-(51). This is a standard situation, so we may leave it aside with respect of what we are interested in here. We retain only the fact that the reflected external field acts upon the dipole, as described by the external-force term in equations (69) and (70). At the same time, the induced dipole generates its own field, as given by equations (56), which acts as a second external field for the half-space; this field polarizes the half-space, which in turn generates a second reflected field, which acts upon the dipole. This is described both in equation (60) and the subsequent equations, and in equations (69). It is essential to realize that this second field depends on the degrees of motion of the dipole, i.e. it is generated by the diplacement field $\mathbf{W}$, as shown in equations (56). Consequently, its effect upon the motion of the dipolar displacemenf field $\mathbf{W}$ is a shift in the eigenfrequencies $\omega_{0}$, which become the "renormalized" frequencies given by equations (65) and (66). These frequencies account for the presence of the half-space; they may bring the dipole response, i.e. the displacement fied $\mathbf{W}$ and the dipole field which governs the Raman scattering, close to resonance, depending on the nature of the dipole (characteristic frequency $\omega_{0}$, plasma frequncy $\omega_{p 0}$ ), the nature of the semi-infinite solid (dielectric function $\varepsilon$ ) and the (relatively small) distance of the dipole to the surface of the semi-infinite solid (equations (65), (66) and (70)).

In order to be specific, we give here the field generated 
by the dipole in the vicinity of the semi-infinite solid, as computed above. This field is given by equations (53) and (54). We limit ourselves to the near-field zone, so we retain only the scalar potential $\Phi_{0}$, with $\lambda=0$; at the same time we assume normal incidence, i.e. we put $i=r=0$ in equations (70) (the general case of oblique incidence can be treated similarly). In this case, there exist only the transverse displacement $\mathbf{w}=\left(w_{1}, w_{2}\right)\left(w_{z}=0\right)$, which we take from equations (70) and introduce in equation (54). By straightforward calculations we get easily

$$
\Phi_{0}(\mathbf{R}, t)=\frac{1}{2 \pi(1+\sqrt{\varepsilon})} \cdot \frac{\omega_{p 0}^{2} v}{\Omega^{2}-\Omega_{\|}^{2}} e^{-i \Omega t}\left(\mathbf{E}_{0} \frac{\partial}{\partial \mathbf{R}}\right) \frac{1}{R}
$$

where

$$
\Omega_{\|} \simeq \omega_{0}-\frac{\omega_{p 0}^{2} v}{64 \pi \omega_{0}} \frac{\varepsilon\left(\omega_{0}\right)-1}{\varepsilon\left(\omega_{0}\right)+1} \cdot \frac{1}{d^{3}}
$$

(according to equation (65)). It is worth noting that $\varepsilon$ in equation (71) is $\varepsilon=\varepsilon(\Omega)$.

First, we note that the field given by equation (71) is a typical dipolar field (the electric field $\mathbf{E}=-\operatorname{grad} \Phi_{0}$ is proportional to $\left.\left[\mathbf{E}_{0} R^{2}-\left(\mathbf{E}_{0} \mathbf{R}\right)^{2}\right] / R^{5}\right)$. Second, we see that the field amplitude in equation (71) is modified by the denominator $\Omega^{2}-\Omega_{\|}^{2}$. For $\Omega$ close to $\Omega_{\|}$we get a resonant behaviour which may account for the enhancement of the Raman effect. The shift in frequency, as given by equation (72), can be either positive or negative, depending on the sign of $\varepsilon\left(\omega_{0}\right)-1$; it is governed mainly by the ratio $v / d$.

The above results, based on equations (70), are valid for a propagating wave. For a damped regime, we must use equation (60) and the field given by equation (23). The calculations are straightforward and we get the damped field of the semi-infinite solid at the position of the dipole

$$
\begin{gathered}
\mathbf{E}_{s r}(\mathbf{r}=0, z=0 ; \omega)= \\
=8 \pi^{2}\left(\frac{i \mathbf{K}}{K}, 1\right) \alpha_{s}(\Omega) E_{0 z} e^{i K_{z} d-K d} \delta(\omega-\Omega),
\end{gathered}
$$

where $\alpha_{s}(\Omega)$ is the surface polarizability given by equation (28). This field enters equations of motion (60) or (70). It is worth noting that it is vanishing for $E_{0 z}=0$ (e.g., an $s$ wave, or normal incidence). We can write now the equation of motion for the displacement field $\mathbf{W}$, which gives access to the response of the dipole placed in the vicinity of a semiinfinite solid, in the damped regime. We limit ourselves to the $z$-component; for an incidence angle $\beta$ we get

$$
\begin{gathered}
\left(\omega^{2}-\Omega_{z}^{2}\right) w_{z}=-\frac{2 \pi q}{m}\left(1+4 \pi i \alpha_{s}(\Omega) e^{i \frac{\Omega}{c} d \cos \beta-\frac{\Omega}{c} d \sin \beta}\right) . \\
\cdot E_{0} \sin \beta \cdot \delta(\omega-\Omega) .
\end{gathered}
$$

Beside the resonance for the shifted frequency $\Omega_{z}$, it is worth noting here the occurrence of the surface polarizability $\alpha_{s}(\Omega)$ given by equation (28), which has a pole for $\omega=\sqrt{\omega_{c}^{2}+\omega_{p}^{2} / 2}$ (surface plasmon frequency) We can see that the response of the dipole can be much enhanced by the presence of the surface plasmons, a typical feature of the surface enhanced Raman scattering.

\section{Conclusions}

The electromagnetic field in matter is described by a new method which includes explicitly the polarization and magnetization degrees of freedom. The Maxwell equations are supplemented by the equations of motion of the polarization and magnetization, thus making possible a full solution. The method is applied to quasi-static fields and the plasmon response of a semi-infinite polarizable (nonmagnetic) solid with a plane surface (half-space). The surface plasmons are highlighted and the surface polarizability is introduced. The electromagnetically coupled system of a neutral, polarizable nano-particle (induced point dipole) and a half-space is solved in the general situation, and the eigenfrequencies and eigenmodes of the zero-point (vacuum) fluctuations are obtained. The van der Waals-LondonCasimir force is computed for this system, as well as its response to an external radiation field, which is the typical problem for the surface enhanced Raman scattering.

\section{Acknowledgement}

The authors are indebted to the members of the Seminar of the Institute of Atomic Physics and the Laboratory of Theoretical Physics, Magurele, Bucharest, for useful discussions and a careful reading of the manuscript. This work was supported by the Romanian Government Research Agency Grant \#306/SMIS 26614.

\section{References}

[1] P. Drude, Zur Elektronentheorie der Metalle, Ann. Phys. 306: 566-613, 1900.

[2] P. Drude, Zur Elektronentheorie der Metalle, 2. Teile. Galvanomagnetische und thermomagnetische Effecte, Ann. Phys. 308: 369-402, 1900.

[3] H. A. Lorentz, The Theory of Electrons, Teubner, Leipzig, 1916.

[4] L. Landau and E. Lifshitz, Course of Theoretical Physics, vol. 2 (The Classical Theory of Fields), Butterworth-Heinemann, Oxford, 2003.

[5] J. Schwinger, L. L. deRaad, Jr., K. A. Milton and W. Tsai, Classical Elctrodynamics, ABP, Westview Press, Boulder, Co., 1998.

[6] J. A. Stratton, Electromagnetic Theory, McGraw-Hill, Cambridge, Mass., 1941.

[7] M. Schwartz, Principles of Electrodynamics, Dover, NY, 1987.

[8] J. D. Jackson, Classical Electrodynamics, Wiley, NJ, 1998.

[9] L. Landau and E. Lifshitz, Course of Theoretical Physics, vol. 8 (Electrodynamics of Continuous Media), Butterworth-Heinemann, Oxford, 2004. 
[10] M. Apostol and G. Vaman, Electromagnetic field interacting with a semi-infinite plasma, J. Opt. Soc. Am. A 26: 1747-1753, 2009.

[11] M. Apostol and G. Vaman, Reflection and refraction of the electromagnetic field in a semi-infinite plasma, Opt. Commun. 282: 4329-4332, 2009.

[12] M. Apostol and G. Vaman, Plasmons and diffraction of an electromagentic plane wave by a metallic sphere, Progr. Electrom., Res. (PIER) 98: 97-118, 2009.

[13] M. Apostol, On the molecular forces acting between macroscopic bodies, Physica B 409: 57-62, 2013.

[14] M. Apostol, Non-inertial electromagnetic effects in matter. Gyromagnetic effect, Solid State Commun. 152: 1567-1571, 2012.

[15] M. Apostol, S. Ilie, A. Petrut, M. Savu and Stefan Toba, A generalization of the dipolar force, J. Appl. Phys. 12: 024905, 2012.

[16] B. F. Apostol, Scattering of the electromagnetic waves from a rough surface, J. Mod. Phys. 59: 1607-1616, 2012.

[17] C. J. F. Bottcher, Theory of Electric Polarisation, Elsevier, Amsterdam, 1952.

[18] P. P. Ewald, Uber die Grundlagen der Kristalloptik, Thesis, Munich, 1912, Ann. Phys. 49(354): 1-38, 1916.

[19] C. W. Oseen, Uber die Wechselwirkung zwischen zwei elektrischen Dipolen der Polarisationsebene in Kristallen und Fluessigkeiten, Ann. Phys. 48(353): 1$56,1915$.

[20] M. Born and E. Wolf, Principles of Optics, Pergamon, London, 1959.

[21] M. Apostol, Essays in Electromagnetism and Matter, Lambert, Saarbrucken, 2013.

[22] Z. Q. Tian, Surface-Enhanced Raman Spectroscopy: It's Present Status, The Internet Journal of Vibrational Spectroscopy 4: ed 2, 2000.

[23] P. Etchegoin, L. F. Cohen, H. Hartigan, R. J. C. Brown, M. J. T. Milton and J. C. Gallop, Electromagnetic contribution to surface enhanced Raman scattering revisited, J. Chem. Phys. 119: 5281-5289, 2003.

[24] M. Moskovits, Surface-Enhanced Raman Spectroscopy: a Brief Perspective, in Surface-Enhanced Raman Scattering, Topics in Applied Physics, vol. 103, pp. 1-17, eds K. Kneipp, M. Moskovits and H. Kneipp, Springer, 2006.

[25] J. R. Lombardi and R. L. Birke, A unified approach to surface-enhanced Raman spectroscopy, J. Phys. Chem. C 112: 5605-5617, 2008.
[26] B. D. Alexander and T. J. Dines, Chemical interactions in the surface-enhanced resonance Raman scattering of ruthenium polypyridyl complexes, J. Phys. Chem. B 109: 3310-3318, 2005.

[27] D. A. Long, The Raman effect: a unified treatment of the theory of Raman scattering by molecules, Wiley, London, 2002.

[28] W. E. Smith and G. Dent, Modern Raman Spectroscopy - A Practical Approach, Wiley, NJ, 2005. 